\title{
Intermediate Coupling For Core-Level Excited States: Consequences For X-Ray Absorption Spectroscopy
}

(Submitted For Volume 200 Issue of J. Electron. Spectros.)

\author{
Paul S. Bagus, ${ }^{a}$ Michel J. Sassi, ${ }^{b}$ and Kevin M. Rosso ${ }^{b}$ \\ ${ }^{\text {a }}$ Department of Chemistry, University of North Texas, Denton, TX 76203-5017, USA \\ ${ }^{\mathrm{b}}$ Pacific Northwest National Laboratory, Richland, WA 99352, USA
}

\begin{abstract}
The origin of the complex NEXAFS features of X-Ray Absorption, XAS, spectra in transition metal complexes is analyzed and interpreted in terms of the angular momentum coupling of the open shell electrons. Especially for excited configurations where a core-electron is promoted to an open valence shell, the angular momentum coupling is intermediate between the two limits of RussellSaunders, RS, coupling where spin-orbit splitting of the electron shells is neglected and $\mathrm{j}-\mathrm{j}$ coupling where this splitting is taken as dominant. The XAS intensities can be understood in terms of two factors: (1) The dipole selection rules that give the allowed excited RS multiplets and (2) The contributions of these allowed multiplets to the wavefunctions of the intermediate coupled levels. It is shown that the origin of the complex XAS spectra is due to the distribution of the RS allowed multiplets over several different intermediate coupled excited levels. The specific case that is analyzed is the $\mathrm{L}_{2,3}$ edge XAS of an $\mathrm{Fe}^{3+}$ cation, because this cation allows a focus on the angular momentum coupling to the exclusion of other effects; e.g., chemical bonding. Arguments are made that the properties identified for this atomic case are relevant for more complex materials. The analysis is based on the properties of fully relativistic, ab initio, many-body wavefunctions for the initial and final states of the XAS process. The wavefunction properties considered include the composition of the wavefunctions in terms of RS multiplets and the occupations of the spin-orbit split open shells; the latter vividly show whether the coupling is $\mathrm{j}-\mathrm{j}$ or not.
\end{abstract}

Keywords: XAS; NEXAFS; Dipole Selection Rules; Intermediate Angular Momentum Coupling

\section{Introduction}

X-Ray Absorption Spectra, XAS, are often taken at edges; i.e., for energies just below and just above ionization limits. In these cases, the spectra have been described as NEXAFS, [1] Near Edge X-Ray Absorption Fine Structure, and we follow this usage. Often, the NEXAFS spectra have several features. For example, for the $\mathrm{L}_{2,3}$, or $2 \mathrm{p}$, edges of transition metal complexes, the NEXAFS have several peaks, rather than simply one each for the $\mathrm{L}_{2}$ and $\mathrm{L}_{3}$ ionization limits; see, for example, the review by de Groot. [2] Furthermore, several distinct final excited states, not always resolved because of broadenings that are due to core- 
hole lifetime and to limits of experimental time and energy resolution, may contribute to an observed spectral feature. [2-3] On the basis of a restricted complete open shell configuration interaction, COSCI, formalism, theoretical predictions for the $\mathrm{L}_{2,3}$ NEXAFS of the isolated $\mathrm{Fe}^{3+}\left(3 \mathrm{~d}^{5} ; \mathrm{S}\right)$ cation are shown in Fig. 1. Here the intensities to individual final states within the configuration $2 \mathrm{p}^{5} 3 \mathrm{~d}^{6}$ have been broadened with a Voigt convolution [4] to represent lifetime and resolution broadening. The details of this spectrum, including the basis of the theoretical predictions, will be described later. For the present our concern is to illustrate the complexity of the spectrum. The dark curve in Fig. 1 is the sum of the contributions from all final states while the underlying curves are from the states making the largest contributions to the total. The leading peak, expected, and shown below, to be from $2 \mathrm{p}_{3 / 2} \rightarrow 3 \mathrm{~d}$ excitations, the $\mathrm{L}_{3}$ edge, has a satellite at $\sim 2 \mathrm{eV}$ higher excitation energy, $\Delta \mathrm{E}$, and less intense satellites at higher $\Delta \mathrm{E}$ of $\sim 6$ to $\sim 10 \mathrm{eV}$. In addition, at higher excitation energies, where the $2 \mathrm{p}_{1 / 2}$ or $\mathrm{L}_{2}$ edge is expected, the total spectrum is a doublet rather than a single peak. Furthermore, judging from the large number of underlying contributions, many final excited states, often unresolved, contribute to the total $\mathrm{L}_{2,3}$ edge XAS curve. As evidence that the theoretical curve of Fig. 1 for the $\mathrm{Fe}^{3+}$ cation includes important physical features for the NEXAFS of ionic complexes, we note that Fig. 1 has significant similarities with the $\mathrm{Mn}_{2,3}$ edge NEXAFS for $\mathrm{MnO}$, where $\mathrm{Mn}^{2+}$ is isoelectronic with $\mathrm{Fe}^{3+}$ both being $3 \mathrm{~d}^{5}$. This is shown by the inset in Fig. 1 where we have digitized and plotted the Mn $2 p \mathrm{~L}_{2,3}$ edge NEXAFS of MnO from Fig. 1(d) in the paper by Bayer et al. [5] We would expect similarities between these two systems since, in both cases, the initial state of the metal cation is $3 \mathrm{~d}^{5}$. Indeed there are similarities between our theory for the $\mathrm{L}_{2,3}$ edge of $\mathrm{Fe}^{3+}$ and the measured $\mathrm{L}_{2,3}$ edge XAS of MnO that we will discuss later in the paper after we have analyzed the origin of the $\mathrm{Fe}^{3+}$ features in our theoretical spectra. However, our main objective in the present paper is to develop an explanation and understanding of the physical origin of the complex features and contributions to the Fe $\mathrm{L}_{2,3}$ edge NEXAFS, Fig. 1 . 
In particular, our concern is show how the concepts of angular momentum coupling and recoupling [6-7] and intermediate coupling [8] lead to the complex NEXAFS spectra; however, we will not use the mathematical formalism of Clebsch-Gordon algebra. [9] An important quantum mechanical principle at the foundation of our analysis is that the final states for core-level ionizations and excitations cannot be represented by a single Slater determinant but must be treated with a many-body formulation. In our, and in many other treatments, the many-body approach involves describing the wavefunctions, WF's, as a summation of Slater determinants or, equivalently, of configuration state functions. [2-3, 1013] A many-body effect that is especially important for NEXAFS is the angular momentum coupling of the open shell electrons. This coupling may be within the open valence, the $3 \mathrm{~d}$ shell of Fe cations, and within the open core shell, the $2 p$ shell for the $\mathrm{L}_{2,3}$ NEXAFS edge, as well as between these shells. When the valence shell coupling changes from the angular momentum coupling in the ground state, we describe this as an angular momentum recoupling. There is also spin-orbit splitting; for the core levels, it is important to distinguish between the $j=\ell+1 / 2$ and the $j=\ell-1 / 2$ spin-orbit split shells. The two limits of angular momentum coupling are Russell-Saunders, RS, coupling [14] where the total orbital angular momentum, $\mathrm{L}$, and the total spin angular momentum, $\mathrm{S}$, are good quantum numbers, and $\mathrm{j}$-j coupling [15] where the spin-orbit splitting dominates. It is common, especially when core holes are present, that neither the RS or $\mathrm{j}$-j limits will dominate and the determinants will mix in such a way that the coupling is intermediate. We will describe both effects of angular momentum recoupling as well as the departures from pure $\mathrm{j}-\mathrm{j}$ coupling as intermediate angular momentum coupling. In the following section, we will discuss the details of the many body effects considered and the criteria that we use to measure the extent of the intermediate coupling. We also consider selection rules for transitions from the ground state to the NEXAFS core-excited final states. In later sections, we apply these measures to characterize the ground and excited 
states and their contributions to the NEXAFS spectra. The concept of the mixing of multiplets in the final NEXAFS excited states is well known and this mixing has been taken into account. This is especially true for treatments that start from non-relativistic solutions and then add spin-orbit interactions; see, for example, Refs. [16-17]. Our work is unique in that we use the presence of multiplets as a physical tool to understand the properties of the excited states rather than primarily as a mathematical tool to obtain wavefunctions for the excited states. [9, 16-17]

In this paper, we consider core excitation in the isolated $\mathrm{Fe}^{3+}$ cation and the $\mathrm{L}_{2,3}$ edge NEXAFS spectra obtained for transitions to these excited states. Fe cations as found in ionic crystals, especially oxides, are important for geochemical processes where the distinction between $\mathrm{Fe}(\mathrm{II})$ and $\mathrm{Fe}(\mathrm{III})$ may be quite important [18-19] and for catalysis where they may often be used as supports; see for example, Ref. [20]. It is clear that the environment of the cations will have an impact on the NEXAFS spectra [2] and that this impact must be taken into account for a predictive understanding of the chemical significance of the features of the NEXAFS spectra. One method that has been used to take the crystalline environment of complexes into account is to represent the crystals with embedded clusters. For a discussion of cluster models of metal complexes used for the analysis of core-level ionization see, for example, Ref. [12] and for the analysis of NEXAFS edges see, for example, Ref. [16-17]. However, there is strong evidence that the methods that we develop to analyze and interpret the spectra of the $\mathrm{Fe}^{3+}$ cation can also be applied for the analysis of materials models that take the oxide environment into account. The main reason that our methods are appropriate for complex systems is that the physical properties that are the basis of these methods, spin-orbit splitting and angular momentum coupling, hold for all point groups. We have chosen to make our first application with atomic symmetry since the analysis is more straightforward and 
simpler with the higher atomic symmetry. [21] This simplicity allows us to tune our theoretical approaches and to explain them in a compact fashion.

The next section of the paper, Sec. II, presents an explanation of the theoretical concepts used to analyze the relativistic wavefunctions in terms of the angular momentum coupling and to determine the intensities of the X-Ray absorption transitions. It also describes the computational methods used. In the following section, "Properties of the initial state of $\mathrm{Fe}^{3+}$, the methods developed in Sec. II are used to show that the lowest, ${ }^{6} \mathrm{~S}_{5 / 2}$, level of $\mathrm{Fe}^{3+}$ is very well described as a RS multiplet. In Sec. IV, "Properties of the final $2 p \rightarrow 3 d$ excited states of $\mathrm{Fe}^{3+\%,}$ the characteristics of these excited states are analyzed. In particular, the relationships between the contribution of the dipole allowed RS multiplet to an intermediate coupled excited level and the NEXAFS intensity of that level is established. Finally, in the last section, our conclusions are summarized

\section{Theoretical Concepts and Computational Methods}

Our methods involve using WF's to describe the initial and final, core-excited states. As we explain below, with this choice angular momentum coupling, especially intermediate coupling, can be treated in a natural fashion. We use four component spinors obtained as solutions of the Dirac Hartree-Fock equations for the average of configurations of the $3 \mathrm{~d}^{5}$ initial, and $2 p^{5} 3 d^{6}$ final, core excited, configurations of $\mathrm{Fe}^{3+}$. [22] (Here, and in the remainder of the paper, we show only the open shells of a configuration.) These spinors are eigenfunctions of $\mathrm{j}^{2}$ and $\mathrm{j}_{\mathrm{z}}$. (Note that we use lower case $\mathrm{j}^{2}$ and $\mathrm{j}_{\mathrm{z}}$ to describe the angular momentum of spinors and upper case $\mathrm{J}^{2}$ and $\mathrm{J}_{\mathrm{Z}}$ to describe the angular momentum of manyelectron wavefunctions.) The use of spinors optimized for an average of configurations makes it possible to determine a large set of wavefunctions with different degeneracies and different 
$\mathbf{J}^{2}$ and $\mathbf{J}_{\mathbf{Z}}$ from a single Hamiltonian matrix, accomplished using the COSCI formalism. [22] For the initial, ground state of $\mathrm{Fe}^{3+}$, determinants are formed with the 5 open shell electrons distributed in all possible ways over the $103 \mathrm{~d}_{3 / 2}$ and $3 \mathrm{~d}_{5 / 2}$ spinors. These determinants are mixed to form many-electron configuration interaction, CI, wavefunctions that are eigenfunctions of the Hamiltonian, $\mathrm{H}$, including $\mathrm{J}^{2}$ and $\mathrm{J}_{\mathrm{z}}$. For this initial state, our concern is for the 6 states that belong to the ground $J=5 / 2$ level. For the final $2 p \rightarrow 3 d$ excited states, determinants are formed by distributing 5 electrons in all possible ways over the $62 \mathrm{p}_{1 / 2}$ and $2 \mathrm{p}_{3 / 2}$ spinors combined with 6 electrons distributed over the $103 \mathrm{~d}$ spinors. As for the initial state, these determinants are mixed in CI WF's to form eigenfunctions of $\mathrm{H}, \mathrm{J}^{2}$ and $\mathrm{J}_{\mathrm{z}}$. However, for these final states, we are interested in all excited states that have non-zero transition probabilities from the initial $\mathrm{J}=5 / 2$ level. All these states contribute to the $\mathrm{L}_{2,3}$ edge NEXAFS spectra. As we demonstrate below, while these states are eigenfunctions of $\mathrm{J}^{2}$ and $\mathrm{J}_{\mathrm{z}}$, their coupling is intermediate between $\mathrm{j}-\mathrm{j}$ and $\mathrm{RS}$ and we directly relate the intermediate coupling with the intensities of excitations to different excited levels.

In order to compare intermediate coupled relativistic solutions with RS coupled multiplets where spin-orbit coupling is neglected, we determine non-relativistic solutions but still using spinors that are eigenfunctions of $\mathrm{j}^{2}$ and $\mathrm{j}_{\mathrm{z}}$. This is done by raising the value of the speed of light. In the limit of $c=\infty$, the exact solution of the Dirac-Hartree-Fock equations reduces to the non-relativistic Hartree-Fock solution where the radial parts of the $j=\ell-1 / 2$ and $j=\ell+1 / 2$ spinors are identical. We have chosen a value $c=7500.0$ atomic units, more than 50 times greater than the correct value of $c=137$ a.u. We then determine orbitals for the same configurations as for the relativistic case. For this value of $c$, the spin-orbit split $2 p$ and $3 d$ orbital energies are the same to within much less than $0.001 \mathrm{eV}$. With these orbitals, the same restricted COSCI many-electron WF's are determined as for the relativistic case. The key feature of this work is that the linear combinations of the determinants now correspond to 
those for the ${ }^{2 S+1} \mathrm{~L}$ non-relativistic RS multiplets. With the large $c=7500$ the energies of the different states in a given multiplet are the same to within a few $\mathrm{meV}$, or often less than an meV. The degeneracies of the orbitals and of the multiplet energies indicate that we have, for this value of $\mathrm{c}$, accurate representations of the non-relativistic solutions.

We obtain transition intensities within the dipole approximation, which leads to selection rules. [23] For levels, where the total orbital and spin angular momentum, J, is the good quantum number, the selections rule is $\Delta \mathrm{J}=0, \pm 1$, where transitions of $\mathrm{J}=0 \rightarrow 0$ are not allowed. However, the transition rules for RS multiplets are stronger. Here the dipole selection rules are $\Delta \mathrm{S}=0$ and $\Delta \mathrm{L}=0, \pm 1$ with $\mathrm{L}=0 \rightarrow 0$ being forbidden. [23] The initial level of $\mathrm{Fe}^{3+}$ is $\mathrm{J}=5 / 2$ and to a very good approximation, see the following section, this level is the RS multiplet ${ }^{6} \mathrm{~S}_{5 / 2}$. [24] Thus the allowed excited levels can be $\mathrm{J}=3 / 2,5 / 2$, and $7 / 2$ while using the multiplet selection rules, the allowed excitations are only to ${ }^{6} \mathrm{P}$ multiplets. This is significant because a $\mathrm{J}=3 / 2$ level can have contributions, among others, from ${ }^{4} \mathrm{P},{ }^{4} \mathrm{D}$, and ${ }^{4} \mathrm{~F}$ multiplets, [14] all of which are dipole forbidden and cannot receive NEXAFS intensity. With the RS multiplets, as determined with a large value of $\mathrm{c}$, it is possible to make a decomposition of the relativistic wavefunctions into the contributions of different multiplets. The particular concern is for the contribution of the dipole allowed ${ }^{6} \mathrm{P}$ multiplet to the different levels. We show in Sec. IV that this contribution provides a useful guide to the intensity of the NEXAFS excitation to a given relativistic level. The contribution of a multiplet is obtained by taking the overlap of the many-electron WF of an RS multiplet with appropriate $\mathbf{J}$ and $\mathrm{J}_{\mathrm{z}}$ with all the many-electron WF's of the levels with the same $\mathrm{J}$ and $\mathrm{J}_{\mathrm{z}}$. The overlap integral for a particular state is normalized with the sum of the overlaps over all the states in the relativistic COSCI. The normalization is required to account for the overlaps between the different orbital sets used for the relativistic and non-relativistic WF's. [25] Here, we represent the contribution of a given RS multiplet as a percentage, $\%$, of the wavefunction 
for the level. These $\%$ contributions are one of the measures that we use for the extent of intermediate coupling in the relativistic WF's; in particular, they show the departures from pure RS multiplets.

Another indication of the extent of intermediate coupling is taken from the occupation numbers of the spin-orbit split pairs of $2 \mathrm{p}_{1 / 2} \& 2 \mathrm{p}_{3 / 2}$ and $3 \mathrm{~d}_{3 / 2} \& 3 \mathrm{~d}_{5 / 2}$ open shell spinors. These occupation numbers, $\mathrm{n}\left(2 \mathrm{p}_{1 / 2}\right), \mathrm{n}\left(2 \mathrm{p}_{3 / 2}\right), \mathrm{n}\left(3 \mathrm{~d}_{3 / 2}\right)$, and $\mathrm{n}\left(3 \mathrm{~d}_{5 / 2}\right)$, are obtained as expectation values of the number operators for the relativistic WF's for the levels; [26] they are the same for all the degenerate states in a given level. If $\mathrm{j}-\mathrm{j}$ coupling holds exactly, these occupation numbers will be integer. For RS and intermediate coupling, the occupations may have fractional values. These occupation numbers are of particular interest for the $2 \mathrm{p}$ shell where the spin-orbit splitting is larger and one expects the occupations in the levels that contribute to the $L_{3}$ edge to be $n\left(2 p_{1 / 2}\right)=2$ and $n\left(2 p_{3 / 2}\right)=3$ while for those that contribute to the $L_{2}$ edge, the occupations will be $n\left(2 p_{1 / 2}\right)=1$ and $n\left(2 p_{3 / 2}\right)=4$. The occupation numbers will be used in Sec. IV to characterize departures from $\mathrm{j}$-j coupling in the $2 \mathrm{p}$ shell.

For NEXAFS intensities, the many-electron dipole matrix elements from the states in the initial $\mathrm{Fe}^{3+} \mathrm{J}=5 / 2$ level to the final, $2 \mathrm{p} \rightarrow 3 \mathrm{~d}$ states of all the levels in the COSCI are calculated. These matrix elements, $\mathrm{ME}_{\mathrm{ij}}(\mathbf{r})$, are

$$
\operatorname{ME}_{\mathrm{ij}}(\mathbf{r})=\left\langle\Psi_{\mathrm{i}}|\mathbf{r}| \Psi_{\mathrm{f}}\right\rangle
$$

where $i$ and $j$ denote individual states of the initial and final level, respectively. We take the NEXAFS intensity to be proportional to the square of the ME, $\mathrm{ME}_{\mathrm{ij}}{ }^{2}$, where we neglect a term in the denominator that depends on the cube of the transition energy. [23] Since the range of energies in the $\mathrm{Fe}^{3+} \mathrm{L}_{2,3}$ edge is $\sim 20 \mathrm{eV}$ and the $2 \mathrm{p} \rightarrow 3 \mathrm{~d}$ excitation energy is $\sim 800 \mathrm{eV}$, it is reasonably accurate to approximate the transition energy as being constant over this energy range. In particular, the concern in NEXAFS spectra is normally for relative intensities, I(rel), 
rather than absolute intensities. Furthermore, sums of $\mathrm{ME}_{\mathrm{ij}}{ }^{2}$ over the degenerate states in the initial and final levels need to be taken to obtain the theoretical NEXAFS shown in Fig. 1. We denote this sum as

$$
\operatorname{ME}_{\mathrm{IJ}}^{2}(\mathbf{r})=\Sigma_{\mathrm{ij}} \mathrm{ME}_{\mathrm{ij}}^{2}(\mathbf{r})
$$

where the summation is taken over the degenerate states, denoted $\mathrm{i}$ and $\mathrm{j}$, in the levels, denoted I and J. The NEXAFS $\mathrm{I}_{\mathrm{IJ}}(\mathrm{rel})$ between the I and $\mathrm{J}$ levels is summed over the Cartesian coordinates,

$$
\mathrm{I}_{\mathrm{IJ}}(\mathrm{rel})=\mathrm{ME}_{\mathrm{IJ}}^{2}(\mathrm{x})+\mathrm{ME}_{\mathrm{IJ}}^{2}(\mathrm{y})+\mathrm{ME}_{\mathrm{IJ}}^{2}(\mathrm{z})
$$

these Cartesian components have the same value given the $\mathrm{S}$ total orbital angular momentum of the initial state of $\mathrm{Fe}^{3+}$. For the plot of Fig. 1, the individual $\mathrm{I}_{\mathrm{IJ}}(\mathrm{rel})$ are broadened by a Voigt convolution [4] of a Lorentzian of $0.5 \mathrm{eV}$ for the lifetime of the $\mathrm{Fe} 2 \mathrm{p}$-hole configuration [27] and a Gaussian of $0.5 \mathrm{eV}$ to represent typical experimental resolutions for the $\mathrm{L}_{2,3}$ edges of $3 \mathrm{~d}$ transition metals. [28] The convoluted $\mathrm{I}_{\mathrm{IJ}}(\mathrm{rel})$ are then summed to yield the full line in Fig. 1 with underlying curves to shown large individual contributions. The $\mathrm{Me}_{\mathrm{ij}}$ are many-electron quantities and cannot be given by a single one-electron matrix element between two spinors because the spinors that are used for the initial and final states are not orthogonal to each other. [25] This is because we take into account the relaxation in response to the core-hole by using a different, relaxed, set of spinors for the final configuration than for the initial configuration. Thus, the $\mathrm{Me}_{\mathrm{ij}}$ involve sums and products of dipole and overlap matrix elements between the spinors optimized for the initial and final configurations. [25]

The basis sets used to describe the Fe cation are uncontracted Gaussians optimized by Wachters [29] and were taken from the EMSL repository. [30] The orbitals, spinors , and wavefunctions were computed with the Dirac program system [31] modified to interface with programs for the calculation of occupation numbers and of many-electron overlap and dipole 
transition matrix elements. These matrix elements were computed using a formalism based on cofactors [32-33] as implemented in the CLIPS program [34] interfaced to Dirac.

\section{Properties of the Initial State of $\mathrm{Fe}^{3+}$}

An immediate measure of the small spin-orbit, SO, interaction in open $3 \mathrm{~d}$ shell of the initial state is that the splitting of the orbital energies of the relativistic $3 \mathrm{~d}_{3 / 2}$ and $3 \mathrm{~d}_{5 / 2}$ DiracHartree-Fock spinors is very small, only $0.15 \mathrm{eV}$. Of course, for the non-relativistic HartreeFock solutions, where the SO interaction is not taken into account, all the d orbitals have exactly the same orbital energy. However, for these non-relativistic $3 \mathrm{~d}$ solutions, we have used a unitary transformation of the standard spin orbitals to spinors that are eigenfunctions of $\mathrm{j}^{2}$ and $\mathrm{j}_{\mathrm{z}}$. This is done, for both the initial and final configurations, to simplify the comparison between the relativistic levels where the SO splitting and coupling of the open shells is taken into account and the RS multiplets, where the SO interaction is neglected. For the initial configuration, the non-relativistic ${ }^{6} \mathrm{~S}$ RS multiplet and the lowest relativistic $\mathrm{J}=5 / 2$ level are compared in Table I. Here, we give the occupations of the $3 d_{3 / 2}$ and $3 d_{5 / 2}$ spinors and the amount of the ${ }^{6} \mathrm{~S}$ multiplet contained in the multiplets of the $\mathrm{J}=5 / 2$ level; we also give the expectation values of the spin and orbital angular momentum, $\left\langle\mathrm{S}_{\mathrm{Z}}\right\rangle$ and $\left\langle\mathrm{L}_{\mathrm{z}}\right\rangle$. The purpose iof these comparisons is to quantify the small departures of the wavefunctions of the $J=5 / 2$ level from those of the $\mathrm{RS}{ }^{56} \mathrm{~S}$ multiplet.

The RS multiplet is, by construction, pure ${ }^{6} \mathrm{~S}$ but the lowest relativistic $\mathrm{J}=5 / 2$ level is almost pure ${ }^{6} \mathrm{~S}$ with $99.9 \%$ character. For the RS multiplet, the occupations of the $3 \mathrm{~d}_{3 / 2}$ and $3 \mathrm{~d}_{5 / 2}$ are exactly 2.0 and 3.0. These occupations do not mean that all determinants in the expansion of the RS multiplet have occupations 2 and 3. It does mean that the weights of the determinants with occupations different from 2 and 3 average to occupations of 3 and 2 . The occupations for the $\mathrm{J}=5 / 2$ level are slightly different from 2.0 and 3.0 consistent with a small 
departure from a pure $\mathrm{RS}{ }^{6} \mathrm{~S}$. Indeed the direction of the departure is to make determinants with occupations larger than 2 have a greater weight in the level than in the RS multiplet. An increase in the average occupation of the $3 \mathrm{~d}_{3 / 2}$ shell is exactly what is expected from the small splitting between the Dirac-Hartree-Fock orbital energies of the $3 \mathrm{~d}_{3 / 2}$ and $3 \mathrm{~d}_{5 / 2}$ spinors. The spin-orbit splitting leads to a $3 \mathrm{~d}_{3 / 2}$ orbital energy that is lower by $0.15 \mathrm{eV}$ than the $3 \mathrm{~d}_{5 / 2}$ orbital energy and this energy lowering favors larger occupation of $3 d_{3 / 2}$. Additional evidence for the nearly pure ${ }^{6} \mathrm{~S}$ character of this level comes from the expectation values of the $\mathrm{z}$-components of the spin and orbital angular momentum, $\left\langle\mathrm{L}_{\mathrm{z}}\right\rangle$ and $\left\langle\mathrm{S}_{\mathrm{Z}}\right\rangle$, for the $\mathrm{J}=5 / 2$ state with the largest values for the projection of the angular momentum on the $\mathrm{z}$-axis. For the pure $\mathrm{RS}{ }^{6} \mathrm{~S}$ multiplet, $\left\langle\mathrm{L}_{\mathrm{z}}\right\rangle=0$ and $\left\langle\mathrm{S}_{\mathrm{z}}\right\rangle=5 / 2$ in units of $\hbar$. For the lowest $\mathrm{J}=5 / 2$ level, the values are $\left\langle\mathrm{L}_{\mathrm{Z}}\right\rangle=0.001$ and $\left\langle\mathrm{S}_{\mathrm{Z}}\right\rangle=2.499$, very small departures from the pure ${ }^{6} \mathrm{~S}$ values.

This gives us the entirely expected result that departures from a pure RS multiplet are very small for the initial state of $\mathrm{Fe}^{3+}\left(3 \mathrm{~d}^{5}\right)$. We will see in the following section that the levels for the excited $2 p \rightarrow 3 d$ core-hole configuration often have much larger departures from either RS or $\mathrm{j}-\mathrm{j}$ coupling and intermediate coupling will be quite important for these core excited states. Furthermore, the near ideal ${ }^{6} \mathrm{~S}$ character of the initial state means that our analysis of the dipole selection rules in terms of transitions being allowed only to final states that have character from the allowed ${ }^{6} \mathrm{P}$ multiplet is valid.

\section{Properties of the Final $2 p \rightarrow 3 d$ excited States of $\mathrm{Fe}^{3+}$}

We begin by considering the lowest 6 levels of the excited state configuration. From the energy expressions for the RS multiplets of the open shell $2 \mathrm{p}^{5} 3 \mathrm{~d}^{6}$ configuration, [14] the lowest multiplet is ${ }^{6} \mathrm{~F}$; this also follows from Hund's rules. This multiplet, which is dipole forbidden will be spin-orbit split into 6 levels with $J=11 / 2$ to $J=1 / 2$. In Table II, we give 
properties of the 6 lowest levels for the excited $2 p \rightarrow 3 d$ configuration. These include the $J$ value of the level, the relative energy with respect to the lowest excited level at $\Delta \mathrm{E}=0$, the $\%$ contributions of the ${ }^{6} \mathrm{~F}$ and the dipole allowed ${ }^{6} \mathrm{P}$ multiplets to the levels. The table also includes the NEXAFS $\mathrm{I}_{\mathrm{IJ}}(\mathrm{rel})$ defined in Eq.(3); we choose to use the simpler notation $\mathrm{I}_{\text {rel }}$ in this table and in the remainder of the paper. The $\mathrm{I}_{\mathrm{rel}}$ in Table II is normalized so the transition to the level with the highest NEXAFS intensity is $I_{\text {rel }}=1$; this level is not included in Table II. The lowest excited level at $\Delta \mathrm{E}=0$ is $\mathrm{J}=11 / 2$ and is $99.9 \%$ a ${ }^{6} \mathrm{~F}$ multiplet; clearly there is little mixing with $\mathrm{J}=11 / 2$ levels arising from other multiplets, for example, ${ }^{4} \mathrm{H},{ }^{4} \mathrm{G}$ and ${ }^{4} \mathrm{I}$, which also exist for the $2 p^{5} 3 d^{6}$ configuration. [14] For $\mathrm{J}=11 / 2$, the first higher energy $\mathrm{J}=11 / 2$ levels are at $\sim 4 \mathrm{eV}$ higher energy; for the other J value levels shown in Table II, other levels of the same $\mathrm{J}$ are at lower energies and these levels are less pure ${ }^{6} \mathrm{~F}$ multiplets. The ${ }^{6} \mathrm{P}$ character for this $\mathrm{J}=11 / 2$ level is exactly zero since the ${ }^{6} \mathrm{P}$ multiplet can only couple to $\mathrm{J}=7 / 2,5 / 2$, and $3 / 2$. Furthermore, the $I_{\text {rel }}$ for this level is exactly zero since a transition from ${ }^{6} \mathrm{~S}_{5 / 2}$ to $\mathrm{J}=11 / 2$ is dipole forbidden. The second level at $\Delta \mathrm{E}=0.6 \mathrm{eV}$ is $\mathrm{J}=9 / 2$ which is also dipole forbidden; it has no ${ }^{6} \mathrm{P}$ character and $\mathrm{I}_{\mathrm{rel}}=0$. It is largely ${ }^{6} \mathrm{~F}$ in character but has almost $30 \%$ from contributions of other multiplets. The third, fourth and fifth levels are dipole allowed $\mathrm{J}=7 / 2$, $5 / 2$, and 3/2, respectively. They have a modest intensity and, in fact, are shown in Fig. 1 as the lowest energy curves contributing to the first NEXAFS peak. The $\mathrm{I}_{\text {rel }}$ given in Table II cannot be directly compared to those in Fig. 1 since the intensity in Fig. 1 is normalized to the maximum of the total intensity summed over the contributions of all final states rather than for Table II where the intensity of a single state has been normalized to 1 . All these three states have some contribution from the ${ }^{6} \mathrm{P}$ multiplet, $\sim 10 \%$, but the $\mathrm{I}_{\text {rel }}$ do not seem to be proportional to the $\%\left({ }^{6} \mathrm{P}\right)$. We shall show below that this is because we have not yet taken into account the fact that these levels have different values of J. Furthermore, the contributions of the ${ }^{6} \mathrm{~F}$ multiplet to these levels is hardly dominant. The ${ }^{6} \mathrm{~F}$ contribution is largest at $47 \%$ for the third level at $\Delta \mathrm{E}=1.1 \mathrm{eV}$ and is quite small, $6.5 \%$, for the fifth level at $\Delta \mathrm{E}=1.8 \mathrm{eV}$. Clearly 
other multiplets make significant contributions to these levels and intermediate coupling is important. The sixth level at $\Delta \mathrm{E}=2.0 \mathrm{eV}$ is $\mathrm{J}=1 / 2$ is dipole forbidden and has no NEXAFS intensity. The very small ${ }^{6} \mathrm{~F}$ character shows that this state has almost no origin from the spinorbit split ${ }^{6} \mathrm{~F}$ multiplet.

Another way of looking at the intermediate angular momentum coupling is shown in Table III, where the $2 p$ and $3 d$ shell occupation numbers are given. The $n\left(2 p_{1 / 2}\right)$ and $n\left(2 p_{3 / 2}\right)$ occupations are very close to the ideal values of 2 and 3 expected for states contributing to the $\mathrm{L}_{3}$ edge NEXAFS. This is hardly surprising since the $13 \mathrm{eV}$ splitting of the Dirac-HartreeFock orbital energies, $\varepsilon$, of the $2 \mathrm{p}_{1 / 2}$ and $2 \mathrm{p}_{3 / 2}$ spinors favors full occupation of the $2 \mathrm{p}_{1 / 2}$ shell for the lower energy excited $2 p \rightarrow 3 d$ states. Thus, the coupling within the $2 p$ shell is dominantly $j-j$ coupling. On the other hand, the occupations of the spin orbit split levels are far from the values of $n\left(3 d_{3 / 2}\right)=4$ and $n\left(3 d_{5 / 2}\right)=2$ expected for $j-j$ coupling for the excited configuration $2 \mathrm{p}^{5} 3 \mathrm{~d}^{6}$. Since the splitting of $\varepsilon\left(3 \mathrm{~d}_{3 / 2}\right)$ and $\varepsilon\left(3 \mathrm{~d}_{5 / 2}\right)$ is only $0.2 \mathrm{eV}$, it is not surprising that Russell Saunders coupling will be important in the $3 \mathrm{~d}$ shell.

The correlation of the NEXAFS $\mathrm{I}_{\text {rel }}$ and the $\%\left({ }^{6} \mathrm{P}\right)$ multiplet character is examined from the data in Tables IV-VI, where these properties are presented separately for the $J=7 / 2$, $5 / 2$, and $3 / 2$ levels, respectively. For each J value, properties are given for the 5 or 6 states with the largest $\mathrm{I}_{\mathrm{rel}}$ for that J. For these states, the relative energy, as in Table II, is given with respect to the lowest energy excited state at $\Delta \mathrm{E}=0$; in this way, the $\Delta \mathrm{E}$ have a consistent meaning over the three tables. The contribution of the ${ }^{6} \mathrm{P}$ multiplet to the levels is given as an absolute contribution, $\%\left({ }^{6} \mathrm{P}\right)$, and a normalized contribution, $\%\left({ }^{6} \mathrm{P}\right)$-norm, with the contribution for the state with the largest $\%\left({ }^{6} \mathrm{p}\right)$ normalized to 1 . The NEXAFS intensities to these levels, $\mathrm{I}_{\mathrm{rel}}$, are also normalized to 1 for the level of this $\mathrm{J}$ value that has the largest intensity. For this normalization, the $\%\left({ }^{6} \mathrm{P}\right)$-norm and the $\mathrm{I}_{\text {rel }}$ can be directly compared. To 
indicate the overall intensity associated with the states of this $\mathrm{J}$ value, the $\mathrm{I}_{\text {rel }}$ normalized to the level with the largest intensity is given in parenthesis for the level with normalized $\mathrm{I}_{\mathrm{rel}}=1$ for each $\mathrm{J}$ value. For the $\mathrm{J}=7 / 2$ levels, 6 levels are given while for the other two $\mathrm{J}$ values only the 5 levels with the largest $\mathrm{I}_{\mathrm{rel}}$ are given. The additional level that is included for $\mathrm{J}=7 / 2$ is at $\Delta \mathrm{E}=1.14 \mathrm{eV}$. This level has a reasonable intensity and it allows there to be one level included in both Tables II and IV.

All the $\mathrm{J}=7 / 2$ levels included in Table IV have reasonable intensities. The least intense level at $\Delta \mathrm{E}=1.1 \mathrm{eV}$ has $30 \%$ of the intensity of the most intense level at $\Delta \mathrm{E}=3.85 \mathrm{eV}$ and it contributes to the low excitation energy part of the first NEXAFS peak; see Fig. 1. The second level at $\Delta \mathrm{E}=2.4 \mathrm{eV}$ contributes to the center of this first peak. The third level at $\Delta \mathrm{E}=3.8 \mathrm{eV}$ contributes to the intense NEXAFS satellite at $\sim 4 \mathrm{eV}$ and the fourth level at $\Delta \mathrm{E}=7.6 \mathrm{eV}$ contributes to the weaker satellite at $\sim 8 \mathrm{eV}$. Finally, the fifth and sixth peaks contribute to the higher excitation energy NEXAFS peaks at $\sim 15$ and $\sim 18 \mathrm{eV}$, respectively. Later, we will examine the extent to which these and the levels with other $\mathrm{J}$ values can be associated with $\mathrm{L}_{2}$ or $\mathrm{L}_{3}$ excitations from the $2 p$ spin-orbit split shells. For the present, we note that the NEXAFS intensity for the $J=7 / 2$ levels is distributed over several excited levels. The least intense of these levels, at $\Delta \mathrm{E}=1.1 \mathrm{eV}$, still has $30 \%$ of the maximum intensity level at $\Delta \mathrm{E}=3.8 \mathrm{eV}$. This is consistent with the fact that the ${ }^{6} \mathrm{P}$ multiplet character is distributed over several levels and the level with the largest amount of ${ }^{6} \mathrm{P}$ character is only $23 \%{ }^{6} \mathrm{P}$. When the ${ }^{6} \mathrm{P}$ character is normalized to 1.0 for the level with largest character, there is excellent agreement with the $\mathrm{I}_{\text {rel }}$ normalized in a similar way. The largest disagreement is for the sixth level with $\Delta \mathrm{E}=17.7 \mathrm{eV}$ and it is only a $6 \%$ difference; see Table IV. Thus, the extent of the NEXAFS allowed ${ }^{6} \mathrm{P}$ multiplet in a $\mathrm{J}=7 / 2$ level provides an excellent guide to the NEXAFS $I_{\text {rel. }}$ This is also true for the other allowed $\mathrm{J}$ values. For the $\mathrm{J}=5 / 2$ levels, the first two levels at 
$\Delta \mathrm{E}=2.1$ and $2.7 \mathrm{eV}$ contribute to the main part of the first NEXAFS peak, the third level at $\Delta \mathrm{E}=4.1 \mathrm{eV}$ contributes to the $\sim 4 \mathrm{eV}$ satellite, and the fourth and fifth levels contribute to the higher energy NEXAFS peaks at $\sim 15$ and $\sim 15 \mathrm{eV}$, respectively. However, the ${ }^{6} \mathrm{P}$ multiplet character for the $\mathrm{J}=5 / 2$ levels is more concentrated into fewer levels with the largest contributions going to the first three levels. For the $\mathrm{J}=5 / 2$ levels, the normalized $\%\left({ }^{6} \mathrm{P}\right)$ also provide an excellent guide to the normalized $I_{\text {rel }}$ with the largest error being $11 \%$ for the relatively low intensity fourth level at $\Delta \mathrm{E}=14 \mathrm{eV}$. For the $\mathrm{J}=3 / 2$ levels, almost all the NEXAFS intensity and almost all the ${ }^{6} \mathrm{P}$ character is in the first two levels at $\Delta \mathrm{E}=1.8$ and 2.4 eV with the largest share going to the second level; see Table VI. The other levels have low $\mathrm{I}_{\mathrm{rel}}$ and little ${ }^{6} \mathrm{P}$ character. Again, the normalized extent of ${ }^{6} \mathrm{P}$ character provides an excellent guide to the $I_{\text {rel }}$ although the errors are a somewhat larger fraction of the $I_{\text {rel }}$ for the low intensity levels at higher excitation energy. The total NEXAFS intensity going into all the levels of the same $\mathrm{J}$ character is calculated to be closely proportional to the degeneracy of the levels and it scales as 8:6:4. This strong dependence of NEXAFS intensity to the degeneracy of the levels explains why the $\%\left({ }^{6} \mathrm{P}\right)$ values shown in Table II did not track the $\mathrm{I}_{\text {rel }}$ especially well since the degeneracy of the different $\mathbf{J}$ values was not taken into account.

Additional properties of the 10 excited levels that make the largest contributions to the $\mathrm{Fe}^{3+} \mathrm{L}_{2,3}$ edge NEXAFS spectra are shown in Table VII. In particular, we show the $2 \mathrm{p}$ shell occupations for these levels in order to determine the extent to which $\mathrm{j}$-j coupling holds for the levels and how accurately the NEXAFS peaks can be separated as either $\mathrm{L}_{3}$ for the lower excitation energy features or $\mathrm{L}_{2}$ for the higher excitation energy features. Furthermore in this table, we give the $\mathrm{I}_{\text {rel }}$ NEXAFS intensities to a common normalization of 1.0 to the most intense peak at $\Delta \mathrm{E}=2.37 \mathrm{eV}$ to allow more direct comparison with the theoretical spectra in Fig. 1. The table includes levels that contribute to all the main NEXAFS peaks in Fig. 1. From the $2 \mathrm{p}$ occupations in Table VII, the main peak and the intense satellite at $\Delta \mathrm{E} \sim 4 \mathrm{eV}$ are 
strongly dominated by determinants where the excitation is from $2 \mathrm{p}_{3 / 2}$; they have less than a $1 \%$ deviation from the ideal $j-j$ coupling values of $n\left(2 p_{1 / 2}\right)=2$ and $n\left(2 p_{3 / 2}\right)=3$. The one level shown that contributes to the less intense satellite at $\Delta \mathrm{E} \sim 8 \mathrm{eV}$ is still dominated by contributions from determinants with excitations from $2 p_{3 / 2}$ but the departures from the ideal values are a bit larger than for the other levels. The two levels, 9 and 10, that contribute to the high excitation energy NEXAFS features, one to each of the doublet, are dominated by determinants that involve excitations from $2 \mathrm{p}_{1 / 2}$ but do involve determinants that have excitations from $2 \mathrm{p}_{3 / 2}$. This is especially true for the level at $\Delta \mathrm{E}=15.29 \mathrm{eV}$ where $\mathrm{n}\left(2 \mathrm{p}_{1 / 2}\right)=1.25$ is a significant departure from the ideal value of $\mathrm{n}\left(2 \mathrm{p}_{1 / 2}\right)=1$ for an $\mathrm{L}_{2}$ edge. Thus, the NEXAFS features can be described as arising dominantly from either $\mathrm{L}_{3}$ or $\mathrm{L}_{2}$ excitations. However, the higher excitation energy $\mathrm{L}_{2}$ features have a larger contamination from $L_{3}$ excitations than the lower energy $L_{3}$ features have through contamination from $L_{2}$ excitations.

The data in Table VII can be used to suggest origins of the features in the experimental $\mathrm{Mn}^{2+} \mathrm{L}_{2,3}$ edge of $\mathrm{MnO}$ [5] shown in the inset of Fig. 1. These suggested assignments are based on the assumption that the $3 \mathrm{~d}^{5}$ open shell of $\mathrm{Fe}^{3+}$ and $\mathrm{Mn}^{2+}$ is a major contributor to the $\mathrm{L}_{2,3}$ edge NEXAFS features. In this spirit, we can associate the first main peak of $\mathrm{MnO}$ at $\sim 640 \mathrm{eV}$ with the three levels, 2-4 in Table VII, of $\mathrm{Fe}^{3+}$ at $\mathrm{E}_{\mathrm{rel}}=2.2$ to $2.36 \mathrm{eV}$. It is also possible to associate the broad feature for $\mathrm{MnO}$ at $\sim 650 \mathrm{eV}$ with levels 9 and 10 in Table VII for $\mathrm{Fe}^{3+}$; in particular, our results indicate that this broad peak arises from atomic multiplet and spin-orbit couplings. In a similar fashion, the other levels in Table VII can also be associated with features of the $\mathrm{MnO}_{2,3}$ edge NEXAFS. The validity of these assignments will be established once we have $\mathrm{L}_{2,3}$ edge calculations and analysis for $\mathrm{Mn}^{2+}$ and for a cluster model of $\mathrm{MnO}$ [7] parallel to those presented here for $\mathrm{Fe}^{3+}$. 


\section{Conclusions and Summary}

We have examined different ways in which the intermediate coupling of the open shell electrons contributes to the core-excited state wavefunctions and, hence, to the $L_{2,3}$ edge NEXAFS spectra of $\mathrm{Fe}^{3+}$. We have shown that this coupling provides a direct explanation of the complex $\mathrm{L}_{2,3}$ edge NEXAFS features that are observed for the NEXAFS of several $3 \mathrm{~d}$ transition metal oxides and other compounds; see, for example, Ref. [2]. While intermediate coupling is important for the $2 \mathrm{p} \rightarrow 3 \mathrm{~d}$ core excited configurations, it is much less important for the initial states where the core shells are filled. Our methods of analysis show that the ground state level of $\mathrm{Fe}^{3+}\left(3 \mathrm{~d}^{5}\right)$ is, to an extremely good approximation, a Russell-Saunders ${ }^{6} \mathrm{~S}$ multiplet. Because of this, it is possible to use the strong dipole selection rule that only transitions from ${ }^{6} \mathrm{~S}$ to ${ }^{6} \mathrm{P}$ are allowed. However the intermediate coupling in the excited levels is generally so important that they cannot be correctly described as Russell-Saunders multiplets. Despite this we have shown that the contribution of a particular Russell-Saunders multiplet to the wavefunctions of any of the excited levels can be determined. This contribution of the dipole allowed ${ }^{6} \mathrm{P}$ multiplet to the wavefunctions of a level gives a simple and reliable guide to the intensity of an XAS excitation to this level. An important consequence of the intermediate coupling in the excited states is that it often leads to a distribution of the ${ }^{6} \mathrm{P}$ multiplet over many different levels. It is this distribution that results in the complex NEXAFS spectra where there are many features. We have explicitly shown that many of the individual contributions to the total NEXAFS intensity cannot be resolved and that a surprisingly large number of individual terms contribute to the total. We have also used the occupations of the spin-orbit split subshells as another way of examining the character of the intermediate angular momentum coupling in the levels of the core-excited configuration. We find that the excitations in the $2 p$ subshells largely follows $j-j$ coupling where the occupations are generally quite close to either $n\left(2 p_{1 / 2}\right)=2$ and $n\left(2 p_{3 / / 2}\right)=3$ for an $L_{3}$ excited 
state or $n\left(2 p_{1 / 2}\right)=1$ and $n\left(2 p_{3 / 2}\right)=4$ for an $L_{2}$ excited state. However, especially for the $L_{2}$ edge levels, there are minor to modest departures from ideal $j-j$ coupling within the $2 p$ shell. On the other hand, the $3 \mathrm{~d}$ shell does not follow $\mathrm{j}$-j coupling. For the initial state, the ${ }^{6} \mathrm{~S}_{5 / 2}$ ground level has each of the spin-orbit split $3 \mathrm{~d}_{3 / 2}$ and $3 \mathrm{~d}_{5 / 2}$ half occupied rather than with the $\mathrm{j}$-j coupling limit of full $n=4$ occupation of $3 d_{3 / 2}$. For the excited levels, the $3 d$ sub-shell occupations remain far from the $\mathrm{j}-\mathrm{j}$ coupling limit but are also not at the limit of occupations found for the Russell-Saunders multiplet coupling of a $3 \mathrm{~d}^{6}$ configuration. This is still another aspect of the intermediate coupling that leads to distribution of the NEXAFS intensity over a large number of states.

Although our novel methods of analysis have been applied to the ideal case of an isolated cation, the same methods can also be applied to cluster models of Fe complexes and compounds as well as for compounds of other metals. We have chosen to make the first application of our new methods to the case of a cation so that the role and interpretation of angular momentum coupling can be examined for a case where this coupling is not modified by the chemical and covalent interactions between the transition metal cation and its ligands. [7] However, for these compounds, there are also theoretical methods that allow us to estimate the extent of their covalent character. [7, 35] By combining the methods we have described here, adjusted to reflect the point group symmetries of the compounds studied, with the previously developed methods to analyze covalency, we are in a position to uniquely characterize the origins of features in the NEXAFS spectra. In particular, we will be able to relate changes in the NEXAFS in different systems to the chemical bonding and interactions in these systems.

\section{Acknowledgements.}


We acknowledge support from the U.S. Department of Energy, Office of Science, Office of Basic Energy Sciences, Chemical Sciences, Geosciences, and Biosciences (CSGB) Division through the Geosciences program at Pacific Northwest National Laboratory.

\section{References}

1. J. Stöhr, NEXAFS Spectroscopy (Springer-Verlag, Berlin, 1992).

2. F. M. F. de Groot, J. Electron Spectrosc. Relat. Phenom. 67, 529 (1994).

3. C. J. Nelin, P. S. Bagus, and E. S. Ilton, RSC Advances 4, 7148 (2014).

4. J. A. Gubner, J. Phys. A 27, L745 (1994).

5. V. Bayer, et al., Phys. Rev. B 76, 165428 (2007).

6. P. S. Bagus, et al., Phys. Rev. Lett. 84, 2259 (2000).

7. P. S. Bagus and E. S. Ilton, Phys. Rev. B 73, 155110 (2006).

8. P. S. Bagus, et al., Chem. Phys. Lett. 546, 58 (2012).

9. G. van der Laan and B. T. Thole, Phys. Rev. B 53, 14458 (1996).

10. K. Okada and A. Kotani, J. Phys. Soc. Jpn. 61, 4619 (1992).

11. M. Taguchi, T. Uozumi, and A. Kotani, J. Phys. Soc. Jpn. 66, 247 (1997).

12. P. S. Bagus, E. S. Ilton, and C. J. Nelin, Surf. Sci. Rep. 68, 273 (2013).

13. M. Roemelt, et al., J. Chem. Phys. 138, 22 (2013).

14. J. C. Slater, Quantum Theory of Atomic Structure, Vols. I \& II (McGraw-Hill, New York, 1960).

15. E. U. Condon and G. H. Shortly, The Theory of Atomic Spectra (Cambridge University Press, Cambridge, 1951).

16. D. Maganas, et al., Phys. Chem. Chem. Phys. 16, 264 (2014).

17. D. Maganas, et al., Phys. Chem. Chem. Phys. 15, 7260 (2013). 
18. K. M. Rosso, D. M. A. Smith, and M. Dupuis, J. Chem. Phys. 118, 6455 (2003).

19. V. Alexandrov and K. M. Rosso, J. Chem. Phys. 140, 234701 (2014).

20. H.-F. Wang, et al., J. Catal. 286, 1 (2012).

21. G. Burns, Introduction to Group Theory With Applications (Academic Press, New York, 1977).

22. L. Visscher, et al., Comput. Phys. Commun. 81, 120 (1994).

23. H. A. Bethe and E. W. Salpeter, Quantum Mechanics of One- and Two-Electron Atoms (Academic Press, 1957).

24. C. E. Moore, Atomic Energy Levels, Natl. Bur. Stand. No. 467, U. S. GPO, Washington, D. C. 1952; see also, URL http://physics.nist.gov/cqi-bin/AtData/main asd.

25. P. O. Löwdin, Phys. Rev. 97, 1474 (1955).

26. L. D. Landau and E. M. Lifshitz, Quantum Mechanics (Addison-Wesley, Reading, 1958).

27. J. L. Campbell and T. Papp, Atomic Data and Nuclear Data Tables 77, 1 (2001).

28. F. P. Netzer, Private Communication.

29. A. J. H. Wachters, J. Chem. Phys. 52, 1033 (1970).

30. The URL for the EMSL basis set data is http://bse.pnl.gov/bse/portal.

31. DIRAC, a relativistic ab initio electronic structure program, Release DIRAC08 (2008), written by L. Visscher, H. J. Aa. Jensen, and T. Saue, with new contributions from R. Bast, S. Dubillard, K. G. Dyall, U. Ekström, E. Eliav, T. Fleig, A. S. P. Gomes, T. U. Helgaker, J. Henriksson, M. Iliaš, Ch. R. Jacob, S. Knecht, P. Norman, J. Olsen, M. Pernpointner, K. Ruud, P. Sałek, and J. Sikkema (see the URL at http://dirac.chem.sdu.dk. .

32. F. Prosser and S. Hagstrom, Int. J. Quantum Chem. 2, 89 (1968).

33. F. Prosser and S. Hagstrom, J. Chem. Phys. 48, 4807 (1968).

34. CLIPS is a program system to compute ab initio SCF and correlated wavefunctions for polyatomic systems. It has been developed based on the publicly available programs in the ALCHEMY package from the IBM San Jose Research Laboratory by P. S. Bagus, B. Liu, A. D. McLean, and M. Yoshimine.

35. P. S. Bagus and C. J. Nelin, J. Electron Spectrosc. Relat. Phenom. 194, 37 (2014). 
Table I. Properties of the lowest $\mathrm{Fe}^{3+}\left(3 \mathrm{~d}^{5}\right)$ states for the non-relativistic RS multiplets, ${ }^{6} \mathrm{~S}(\mathrm{RS})$, and the relativistic $J=5 / 2$ level. The percent ${ }^{6} S$ character and the occupation numbers, $n\left(3 d_{3 / 2}\right)$ and $n\left(3 d_{5 / 2}\right)$, are given. Also given are the expectation values of the orbital and spin angular momentum, $\left\langle\mathrm{L}_{\mathrm{z}}\right\rangle$ and $<\mathrm{S}_{\mathrm{Z}}>$

\begin{tabular}{|l|l|l|l|l|l|}
\hline & $\%\left({ }^{6} \mathrm{~S}\right)$ & $\mathrm{n}\left(\left(3 \mathrm{~d}_{3 / 2}\right)\right.$ & $\mathrm{n}\left(\left(3 \mathrm{~d}_{5 / 2}\right)\right.$ & $\left\langle\mathrm{S}_{\mathrm{z}}\right\rangle$ & $\left\langle\mathrm{L}_{\mathrm{z}}\right\rangle$ \\
\hline${ }^{6} \mathrm{~S}(\mathrm{RS})$ & 100.0 & 2.00 & 3.00 & 2.500 & 0.000 \\
\hline $\mathrm{J}=5 / 2$ & 99.9 & 2.05 & 2.95 & 2.499 & 0.001 \\
\hline
\end{tabular}

Table II. Properties of the 6 lowest excited $2 p \rightarrow 3 d$ levels. The properties include the relative energy of the levels, $\Delta \mathrm{E}$, with $\Delta \mathrm{E}=0.0$ for the lowest level, the $\mathrm{J}$ value of the level, the ${ }^{6} \mathrm{~F}$ and ${ }^{6} \mathrm{P}$ multiplet character, and the relative NEXAFS intensity, $I_{r e l}$, The $I_{\text {rel }}$ is normalized so that the level with the largest intensity, not shown in this table, has $\mathrm{I}_{\mathrm{rel}}=1$. See caption to Table I.

\begin{tabular}{|l|l|l|l|l|l|}
\hline & $\Delta \mathrm{E}-\mathrm{eV}$ & $\mathrm{J}$ & $\%\left({ }^{6} \mathrm{~F}\right)$ & $\%\left({ }^{6} \mathrm{P}\right)$ & $\mathrm{I}_{\text {rel }}$ \\
\hline 1 & 0.00 & $11 / 2$ & 99.9 & --- & 0.00 \\
\hline 2 & 0.58 & $9 / 2$ & 72.6 & --- & 0.00 \\
\hline 3 & 1.14 & $7 / 2$ & 46.5 & 7.1 & 0.19 \\
\hline 4 & 1.50 & $5 / 2$ & 18.2 & 7.4 & 0.14 \\
\hline 5 & 1.81 & $3 / 2$ & 6.5 & 10.2 & 0.13 \\
\hline 6 & 2.01 & $1 / 2$ & 1.4 & ---- & 0.00 \\
\hline
\end{tabular}


Table III. Open shell occupation numbers for the 6 lowest excited $2 p \rightarrow 3 \mathrm{~d}$ levels.

\begin{tabular}{|l|l|l|l|l|}
\hline & $\mathrm{n}\left(2 \mathrm{p}_{1 / 2}\right)$ & $\mathrm{n}\left(2 \mathrm{p}_{3 / 2}\right)$ & $\mathrm{n}\left(3 \mathrm{~d}_{3 / 2}\right)$ & $\mathrm{n}\left(3 \mathrm{~d}_{5 / 2}\right)$ \\
\hline 1 & 2.00 & 3.00 & 2.85 & 3.15 \\
\hline 2 & 2.00 & 3.01 & 2.44 & 3,56 \\
\hline 3 & 1.99 & 3.01 & 2.19 & 3.81 \\
\hline 4 & 1.99 & 3.01 & 2.40 & 3.60 \\
\hline 5 & 1.99 & 3.01 & 2.30 & 3.70 \\
\hline 6 & 1.99 & 3.01 & 2.39 & 3.61 \\
\hline
\end{tabular}

Table IV. Properties of the 6 lowest excited $2 p \rightarrow 3 d$ levels that have $J=7 / 2$ degeneracy. Properties include relative energy, $\Delta \mathrm{E}$ with respect to the lowest excited level, $\Delta \mathrm{E}=0$, the $\%\left({ }^{6} \mathrm{P}\right)$ multiplet character and the intensity of the excited levels, $\mathrm{I}_{\text {rel }}$. The $\%\left({ }^{6} \mathrm{P}\right)$-norm and the $\mathrm{I}_{\text {rel }}$ are normalized so that the level with the largest $\%\left({ }^{6} \mathrm{P}\right)$ and $\mathrm{I}_{\text {rel }}$ are both normalized to 1.0 . For the largest $\mathrm{I}_{\mathrm{rel}}$, the value, in parenthesis, is with respect to the largest intensity over all the excited states normalized to 1.0.

\begin{tabular}{|l|l|l|l|l|}
\hline & $\Delta \mathrm{E}-\mathrm{eV}$ & $\%\left({ }^{6} \mathrm{P}\right)$ & $\%\left({ }^{6} \mathrm{P}\right)$-norm & $\mathrm{I}_{\text {rel }}$ \\
\hline 1 & 1.14 & 7.1 & 0.31 & 0.31 \\
\hline 2 & 2.36 & 13.7 & 0.60 & 0.60 \\
\hline 3 & 3.85 & 23.0 & 1.00 & $1.00(0.60)$ \\
\hline 4 & 7.65 & 9.8 & 0.43 & 0.43 \\
\hline 5 & 15.29 & 9.2 & 0.40 & 0.38 \\
\hline 6 & 17.75 & 17.4 & 0.76 & 0.71 \\
\hline
\end{tabular}


Table V. Properties of the 5 lowest excited $2 p \rightarrow 3 d$ levels that have $J=5 / 2$ degeneracy; see caption to Table IV.

\begin{tabular}{|l|l|l|l|l|}
\hline & $\Delta \mathrm{E}-\mathrm{eV}$ & $\%\left({ }^{6} \mathrm{P}\right)$ & $\%\left({ }^{6} \mathrm{P}\right)$-norm & $\mathrm{I}_{\text {rel }}$ \\
\hline 1 & 2.12 & 40.0 & 1.00 & $1.00(0.80)$ \\
\hline 2 & 2.69 & 12.2 & 0.30 & 0.31 \\
\hline 3 & 4.12 & 12.3 & 0.30 & 0.31 \\
\hline 4 & 14.84 & 7.1 & 0.16 & 0.18 \\
\hline 5 & 17.56 & 5.8 & 0.13 & 0.13 \\
\hline
\end{tabular}

Table VI. Properties of the 5 lowest excited $2 p \rightarrow 3 d$ levels that have $\mathrm{J}=3 / 2$ degeneracy; see caption to Table IV.

\begin{tabular}{|l|l|l|l|l|}
\hline & $\Delta \mathrm{E}-\mathrm{eV}$ & $\%\left({ }^{6} \mathrm{P}\right)$ & $\%\left({ }^{6} \mathrm{P}\right)$-norm & $\mathrm{I}_{\text {rel }}$ \\
\hline 1 & 1.81 & 10.2 & 0.13 & 0.13 \\
\hline 2 & 2.37 & 76.0 & 1.00 & $1.00(1.00)$ \\
\hline 3 & 4.39 & 2.2 & 0.03 & 0.03 \\
\hline 4 & 14.84 & 4.5 & 0.06 & 0.05 \\
\hline 5 & 16.51 & 1.7 & 0.02 & 0.02 \\
\hline
\end{tabular}


Table VII. Properties of the 10 lowest excited $2 p \rightarrow 3 d$ levels. The properties include the relative energy of the levels, $\Delta E$, with $\Delta E=0.0$ for the lowest level, the $J$ value of the level, the $n\left(2 p_{1 / 2}\right)$ and $n\left(2 p_{3 / 2}\right)$ occupations of the spin-orbit split $2 p$ shells, and the relative intensities, $I_{\text {rel, }}$ of levels; see text and caption to Table I.

\begin{tabular}{|c|l|l|l|l|l|}
\hline & $\Delta \mathrm{E}-\mathrm{eV}$ & $\mathrm{J}$ & $\mathrm{n}\left(2 \mathrm{p}_{1 / 2}\right)$ & $\mathrm{n}\left(2 \mathrm{p}_{3 / 2}\right)$ & $\mathrm{I}_{\text {rel }}$ \\
\hline 1 & 1.14 & $7 / 2$ & 1.99 & $3 / 01$ & 0.19 \\
\hline 2 & 2.12 & $5 / 2$ & 2.00 & 3.00 & 0.80 \\
\hline 3 & 2.36 & $7 / 2$ & 1.98 & 3.02 & 0.36 \\
\hline 4 & 2.37 & $3 / 2$ & 2.00 & 3.00 & 1.00 \\
\hline 5 & 2.69 & $5 / 2$ & 1.97 & 3.03 & 0.24 \\
\hline 6 & 3.85 & $7 / 2$ & 1.99 & 3.01 & 0.60 \\
\hline 7 & 4.12 & $5 / 2$ & 1.97 & 3.03 & 0.24 \\
\hline 8 & 7.65 & $7 / 2$ & 1.94 & 3.06 & 0.26 \\
\hline 9 & 15.29 & $7 / 2$ & 1.25 & 3.75 & 0.23 \\
\hline 10 & 17.75 & $7 / 2$ & 1.11 & 3.98 & 0.43 \\
\hline
\end{tabular}


Fig. 1. Theoretical $\mathrm{L}_{2,3}$ edge NEXAFS for the $\mathrm{Fe}^{3+}$ cation. The full line is the sum of contributions to the NEXAFS from all allowed final states and the underlying lines (in color online) are for individual contributions of final states with large intensities. The zero of energy is taken as the energy of the lowest level of the excited ......2 $\mathrm{p}^{5} \ldots \ldots .3 \mathrm{~d}^{6}$ configuration. The Voigt broadening and other details are described in Sec. IV of the text. The inset for the experimental $\mathrm{L}_{2,3}$ NEXAFS edge of MnO is plotted from the data in Ref. [5]

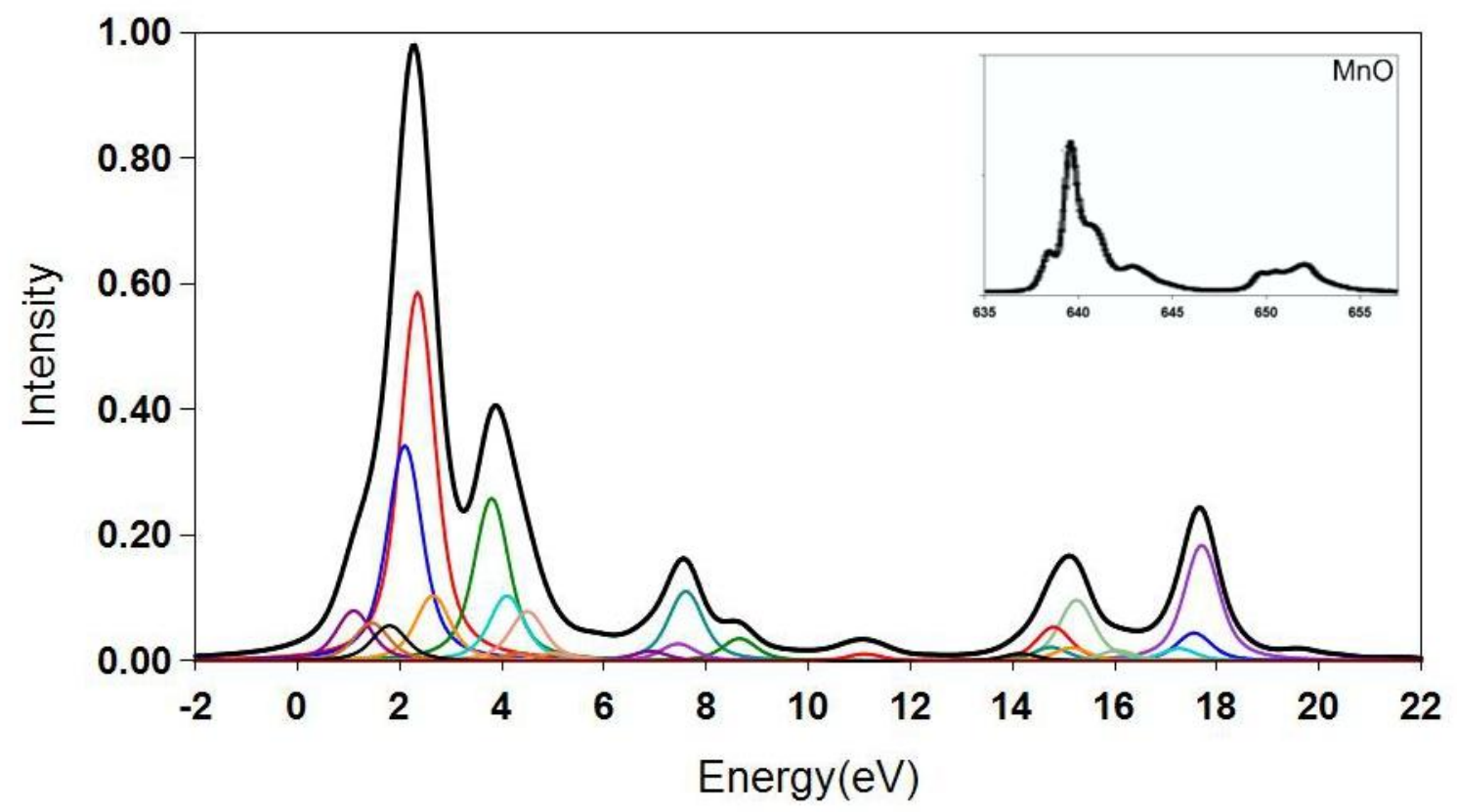

